\title{
Mercaptopurine Anhydrous
}

National Cancer Institute

\section{Source}

National Cancer Institute. Mercaptopurine Anhydrous. NCI Thesaurus. Code C61832.

The anhydrous form of mercaptopurine, a thiopurine-derivative antimetabolite with antineoplastic and immunosuppressive activities. Produced through the metabolism of mercaptopurine by hypoxanthine-guanine phosphoribosyltransferase (HGPRT), mercaptopurine metabolites 6-thioguanosine-5'-phosphate (6-thioGMP) and 6thioinosine monophosphate (T-IMP) inhibit nucleotide interconversions and de novo purine synthesis, thereby blocking the formation of purine nucleotides and inhibiting DNA synthesis. This agent is also incorporated into DNA in the form of deoxythioguanosine, which results in the disruption of DNA replication. In addition, mercaptopurine is converted to 6-methylmercaptopurine ribonucleoside (MMPR) by 6-thiopurine methyltransferase; MMPRs are also potent inhibitors of de novo purine synthesis. 